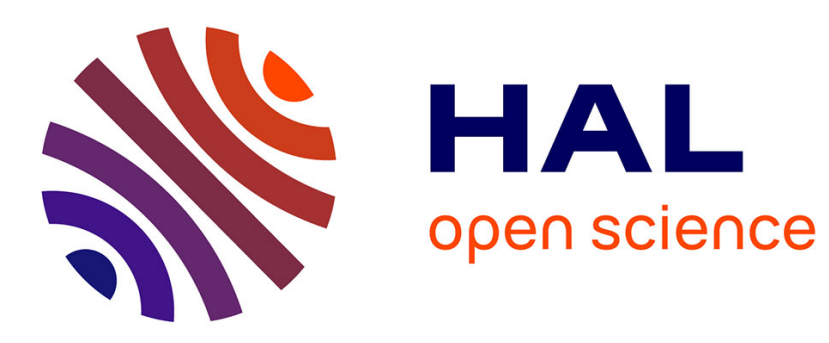

\title{
La vertu politique comme principe de la démocratie. Robespierre lecteur de Montesquieu \\ Céline Spector
}

\section{To cite this version:}

Céline Spector. La vertu politique comme principe de la démocratie. Robespierre lecteur de Montesquieu. Michel Biard; Philippe Bourdin; Hervé Leuwers; Alain Tourret. Vertu et Politique. Les pratiques des législateurs (1789-2014), Presses universitaires de Rennes, pp.61-70, 2015, 978-2-75354138-2. hal-02475992

\section{HAL Id: hal-02475992 \\ https://hal.sorbonne-universite.fr/hal-02475992}

Submitted on 13 Feb 2020

HAL is a multi-disciplinary open access archive for the deposit and dissemination of scientific research documents, whether they are published or not. The documents may come from teaching and research institutions in France or abroad, or from public or private research centers.
L'archive ouverte pluridisciplinaire HAL, est destinée au dépôt et à la diffusion de documents scientifiques de niveau recherche, publiés ou non, émanant des établissements d'enseignement et de recherche français ou étrangers, des laboratoires publics ou privés. 


\title{
La vertu politique comme principe de la démocratie Robespierre lecteur de Montesquieu
}

\author{
Céline Spector
}

\begin{abstract}
«La plupart des peuples anciens vivaient dans des gouvernements qui ont la vertu pour principe; et, lorsqu'elle y était dans sa force, on y faisait des choses que nous ne voyons plus aujourd'hui, et qui étonnent nos petites âmes. »
\end{abstract}

De l'esprit des lois, IV, 4.

Dans son célèbre discours du 17 pluviôse an II-5 février 1794, Robespierre définit le principe fondamental du gouvernement "démocratique ou populaire » :

"La démocratie est un état où le peuple souverain, guidé par des lois qui sont son ouvrage, fait par lui-même tout ce qu'il peut bien faire, et par des délégués tout ce qu'il ne peut faire lui-même.

C'est donc dans les principes du gouvernement démocratique que vous devez chercher les règles de votre conduite politique [...].

Or, quel est le principe fondamental du gouvernement démocratique ou populaire, c'est-à-dire le ressort essentiel qui le soutient et qui le fait mouvoir? C'est la vertu; je parle de la vertu publique qui opéra tant de prodiges dans la Grèce et dans Rome, et qui doit en produire de bien plus étonnants dans la France républicaine; de cette vertu qui n'est autre chose que l'amour de la patrie et de ses lois.

Mais comme l'essence de la république ou de la démocratie est l'égalité, il s'ensuit que l'amour de la patrie embrasse nécessairement l'amour de l'égalité. Il est vrai encore que ce sentiment sublime suppose la préférence de l'intérêt public à tous les intérêts particuliers; d'où il résulte que l'amour de la patrie suppose encore ou produit toutes les vertus: car que sont-elles autre chose que la force de l'âme qui rend capable de ces sacrifices? Et comment l'esclave de l'avarice ou de l'ambition, par exemple, pourrait-il immoler son idole à la patrie? Non seulement la vertu est l'âme de la démocratie; mais elle ne peut exister que dans ce gouvernement ${ }^{1} \ldots$ ”

1. Robespierre, Euvres complètes (désormais OC), t.X, p. 353. Les Euvres en dix volumes ont été publiées par la Société des Études Robespierristes entre 1910 et 1967, puis complétées par Florence Gauthier en 2007. 
Or cet éloge de la vertu a une source précise: Robespierre emprunte ici directement à Montesquieu, plus encore qu'à Rousseau. Son discours compile des textes issus du livre II (II, 2), du livre III (III, 3) et du livre V (V, 2-5) de l'Esprit des lois. Comme Montesquieu, Robespierre fait de la démocratie une espèce de république dont le "principe " ou la passion dominante est la vertu, c'est-à-dire l'amour de la patrie, l'amour des lois et de l'égalité. La seule prise de distance à l'égard de Montesquieu dans ce passage est remarquable: "Je parle de la vertu publique qui opéra tant de prodiges dans la Grèce et dans Rome, et qui doit en produire de bien plus étonnants dans la France républicaine" (n. s.). Montesquieu n'avait évidemment pas envisagé cette possibilité, lui qui croyait que la vertu, désormais, était hors d'atteinte pour les modernes, et que la démocratie était, pour l'essentiel, chose du passé2. C'est pourtant à Montesquieu, plus encore qu'à Rousseau, que Robespierre se réfère, de manière explicite, lorsqu'il évoque l'amour des lois dès son Discours adressé à messieurs de la société littéraire de Metz du 25 août 1784 :

"Le ressort essentiel des républiques, est la vertu, comme l'a prouvé l'auteur de l'esprit des loix, c'est-à-dire la vertu politique, qui n'est autre chose que l'amour des loix et de la patrie: leur constitution même érige que tous les intérêts particuliers, toutes les liaisons personnelles cèdent sans cesse au bien général ${ }^{3}$."

Cette contribution se propose de montrer en quoi la défense de la cause du peuple et de la régénération de la nation conduit pourtant Robespierre à s'éloigner in fine des principes de l'un de ses mentors. L'Esprit des lois est sans conteste l'une des œuvres majeures dans la formation intellectuelle de Robespierre, comme elle l'a été pour de nombreux artisans de la Révolution, comme Marat ou Saint-Just ${ }^{4}$. Il convient donc de se demander à quelles conditions Robespierre a pu contrer les mises en garde de Montesquieu sur l'impossibilité de la vertu chez les modernes, dans le contexte singulier d'une consolidation de la Révolution contre la menace de rechute dans la tyrannie.

2. Voir Althusser L., Montesquieu. La politique et l'histoire, Paris, PUF, 1959.

3. OC, t. I, p. 24. Voir BOSC Y., « Robespierre et l'amour des lois », Jus Politicum, n 10 [http://www. juspoliticum.com/Robespierre-et-l-amour-des-lois.html]; VAN DER HALLEN TH., "Corruption et régénération du politique chez Robespierre ", Anabases, n ${ }^{6}$, 2007, p. 67-82 (en ligne).

4. Sur les usages (modérés, réactionnaires ou révolutionnaires) de Montesquieu pendant la Révolution, voir Manin B., "Montesquieu ", dans Furet F. et Ozouf M. [dir.], Dictionnaire critique de la Révolution française, Paris, Flammarion, 1988; Roger BARNY, "Montesquieu dans la Révolution française ", Annales historiques de la Révolution française, 1990, p. 48-73; Barny R., "Montesquieu patriote? ", Ehrard J. et Benrekassa G. [dir.], numéro spécial Montesquieu et la Révolution, Dix-Huitième Siècle 21, Paris, PUF, 1989, p. 83-95; BART J., "Révolution française ", dans Dictionnaire Montesquieu [en ligne], Catherine Volpilhac-Auger [dir.], ENS Lyon, septembre 2013 [http://dictionnaire-montesquieu. ens-lyon.fr/fr/article/1367163329/fr]. Le jeune Saint-Just, en 1791, publie L'Esprit de la Révolution et de la Constitution de France avec, en épigraphe, une phrase de L'Esprit des lois. Marat, dans son Projet de Déclaration des droits de l'homme et du citoyen suivi d'un Plan de constitution juste, sage et libre d'août 1789, insère un dithyrambe de Montesquieu qu'il considère comme l'un de ses maittres. 
Un dernier préalable. Il va de soi, que Robespierre n'a pas été un Montesquieu, moins encore qu'un Rousseau " au pouvoir ", dossier encore brûlant que nous ne rouvrirons ici que de façon très marginale. Robespierre construit, en fonction d'un contexte en constante évolution, une trajectoire politique qui lui est propre. Nous souhaiterions donc mettre à jour de véritables structures de pensées, qui ne sont certes pas projetées comme un a priori philosophique sur la matière ductile de l'histoire, mais conçues sous formes de "principes " et adaptées, sous formes de maximes, aux circonstances hic et nunc.

\section{La vertu politique, principe de la démocratie}

Dans L'Esprit des lois, Montesquieu propose une typologie des régimes politiques (démocratie ou aristocratie, monarchie, despotisme) et fait correspondre à leur «nature " (leurs institutions) un "principe », ressort ou passion dominante, qui leur permet de persévérer dans l'être sans corruption ni révolution: vertu ou modération, honneur, crainte ${ }^{5}$. Ainsi dans les démocraties, où chaque citoyen est à la fois souverain et sujet, où « le peuple en corps a la souveraine puissance » (II, 2), les citoyens doivent être vertueux, sans quoi le gouvernement sera "imparfait» (III, 9).

Pourquoi la vertu? La difficulté spécifique de la démocratie tient au rapport du citoyen aux lois : l'auteur des lois sera aussi leur sujet. Si l'obéissance ne requiert que la « force des lois » en monarchie, si elle n'exige que la force brute dans le despotisme, la démocratie est ce régime où l'auteur des lois est en même temps celui qui doit faire exécuter les lois et en porter le " poids" (III, 3). La vertu permet de surmonter ce problème de l'autocontrainte: seuls des citoyens mus par l'amour des lois sont prêts à préférer l'intérêt général à leurs intérêts particuliers. La dimension d'attachement affectif impliquée par le terme d'amour revêt ici une importance capitale: la vertu fournit au citoyen la motivation nécessaire pour le respect de la règle alors même que celle-ci va à l'encontre de ses intérêts et de ses passions égoïstes (avarice, ambition) : "On peut définir cette vertu, l'amour des lois et de la patrie. Cet amour, demandant une préférence continuelle de l'intérêt public au sien propre, donne toutes les vertus particulières; elles ne sont que cette préférence " (IV, 5). Chaque citoyen doit éprouver un "zèle sans bornes" pour le bien public (VI, 8).

\footnotetext{
5. Voir Spector C., Montesquieu. Liberté, droit et histoire, Paris, Michalon, 2010 ; Larrère C., "Droit et mœurs chez Montesquieu ", dans Hoquet T. et Spector C. [dir.], Lectures de L'Esprit des lois, Bordeaux, Presses Universitaires de Bordeaux, 2004, p. 233-246; Larrère C., " Montesquieu et l'exception française ", dans Domenico Felice [dir.], Poteri, Democrazia, Virtù. Montesquieu nei movimenti repubblicani alléepoca della Rivoluzione francese, Milan, FrancoAngeli, 2000, p. 51-64. Nous citerons L'Esprit des lois, Derathé R. éd., De Casabianca D. rééd., Paris, Classiques Garnier, 2011, en précisant simplement le livre et le chapitre.
} 
Trois remarques s'imposent ici : en premier lieu, la vertu politique ainsi définie comme vertu civique n'est ni la vertu morale, ni la vertu chrétienne. L'Avertissement ajouté à l'édition posthume de 1757, destiné à répondre aux critiques des censeurs, est très clair:

"Pour l'intelligence des quatre premiers livres de cet ouvrage, il faut observer que ce que j'appelle la vertu dans la république est l'amour de la patrie, c'est-à-dire l'amour de l'égalité. Ce n'est point une vertu morale, ni une vertu chrétienne; c'est la vertu politique [...] J'ai eu des idées nouvelles; il a bien fallu trouver de nouveaux mots, ou donner aux anciens de nouvelles acceptions ".

En second lieu, la vertu est décrite comme une passion et non comme l'effet de la raison. La vertu est une passion politique, la passion démocratique, apanage du peuple et non privilège d'une élite:

"La vertu, dans une république, est une chose très simple: c'est l'amour de la république; c'est un sentiment, et non une suite de connaissances; le dernier homme de l'État peut avoir ce sentiment, comme le premier. Quand le peuple a une fois de bonnes maximes, il s'y tient plus longtemps que ce qu'on appelle les honnêtes gens. Il est rare que la corruption commence par lui. Souvent il a tiré de la médiocrité de ses lumières un attachement plus fort pour ce qui est établi $(\mathrm{V}, 2)$. "

Enfin, l'amour de la patrie et l'amour des lois supposent également, selon Montesquieu, l'amour de l'égalité et de la frugalité $(\mathrm{V}, 3)$, qui doivent être soutenues par l'existence de l'égalité et de la frugalité elles-mêmes: dans les démocraties, "c'est à des lois particulières à égaliser, pour ainsi dire, les inégalités, par les charges qu'elles imposent aux riches, et le soulagement qu'elles accordent aux pauvres" $(\mathrm{V}, 5)$. L'Esprit des lois ne se contente donc pas de faire de la vertu le principe de la démocratie: il démocratise la vertu elle-même afin de la décerner au peuple tout entier, et non plus aux meilleurs - à une aristocratie de l'excellence.

Mais cette démocratisation de la vertu a un prix. La vertu n'est pas le privilège de naissance d'une élite, ni l'apanage d'être supérieurs; elle est difficile pour tout le monde. Montesquieu pose donc la question de la métamorphose de l'homme en citoyen: alors que «l'honneur des monarchies est favorisé par les passions, et les favorise à son tour ", la vertu politique " est un renoncement à soi-même, qui est toujours une chose très pénible "; elle ne peut donc se soutenir que grâce à la toute-puissance de l'éducation, lorsqu'un peuple se considère comme une seule famille (IV, 5). Montesquieu va jusqu'à prendre l'image du monastère pour illustrer le transfert requis des passions égoïstes (cupidité, ambition) vers une passion unique - celle du général et de la règle:

"Moins nous pouvons satisfaire nos passions particulières, plus nous nous livrons aux générales. Pourquoi les moines aiment-ils tant leur ordre? 
C'est justement par l'endroit qui fait qui leur est insupportable. Leur règle les prive de toutes les choses sur lesquelles les passions ordinaires s'appuient: reste donc cette passion pour la règle même qui les afflige. Plus elle est austère, c'est-à-dire, plus elle retranche de leurs penchants, plus elle donne de force à ceux qu'elle leur laisse $(\mathrm{V}, 2)$. "

Telle est la grande découverte de Montesquieu: la vertu repose sur une forme de dénaturation ${ }^{6}$. À cet égard, la démocratie ne peut se conserver par la seule force des lois qui garantissent l'existence de l'égalité et de la frugalité (lois agraires qui divisent les terres, lois somptuaires qui répriment le luxe, lois de succession qui assurent le partage des richesses). La république suppose des institutions chargées de la surveillance des mœurs. Elle suppose des censeurs, gardiens de la vertu, chargés du " dépôt des mœurs: il faut que les censeurs, comme à Rome, "rétablissent dans la république tout ce qui a été corrompu, qu'ils notent la tiédeur, qu’ils jugent les négligences et corrigent les fautes, comme les lois punissent les crimes " $(\mathrm{V}, 7)$. En d'autres termes, la conservation des démocraties exige l'entretien d'une morale publique extrêmement rigoureuse:

"Ce ne sont pas seulement les crimes qui détruisent la vertu, mais encore les négligences, les fautes, une certaine tiédeur dans l'amour de la patrie, des exemples dangereux, des semences de corruption; ce qui ne choque point les lois, mais les élude, ce qui ne les détruit pas, mais les affaiblit » $(\mathrm{V}, 19)$.

\section{De la corruption en démocratie: Montesquieu et les infortunes de la vertu}

Parce que les forces de l'histoire agissent dans le sens du déclin des corps politiques, la question de la corruption est originaire pour Montesquieu ${ }^{7}$. Dans L'Esprit des lois, le terme de corruption apparaît pour la première fois à propos de la passion dominante des démocraties:

"Lorsque, dans un gouvernement populaire, les lois ont cessé d'être exécutées, comme cela ne peut venir que de la corruption de la république, l'État est déjà perdu " (III, 3).

Par-là, Montesquieu établit le primat du principe dans l'histoire:

"Lorsque les principes du gouvernement sont une fois corrompus, les meilleures lois deviennent mauvaises, et se tournent contre l'État; lorsque

6. Voir Manent P., La Cité de l'homme, Paris, Champs Flammarion, 1995, chapitre 2. Cette lecture, partagée par d'autres (Thomas Pangle, Paul Rahe) est celle de Leo Strauss dans ses séminaires de 1965-1966 à l'université de Chicago. Voir Spector C., "Montesquieu et la crise du droit naturel moderne. L'exégèse straussienne ", Revue de Métaphysique et de Morale, numéro spécial sur "Montesquieu ", de Casabianca D. et Mennasseyre Ch. [dir.], 2013 (1), p. 65-78.

7. Voir Spector C., "Corruption ", dans Dictionnaire Montesquieu [en ligne], VolpilhacAuger C. [dir.], ENS Lyon, septembre 2013 [http://dictionnaire-montesquieu.ens-lyon.fr/fr/ article/1376473889/fr/]. 
les principes en sont sains, les mauvaises ont l'effet des bonnes; la force du principe entraîne tout " (VIII, 11).

À ce titre, la démocratie est en situation particulièrement délicate: elle subit le risque constant d'une corruption de la vertu. Le risque est double, car la vertu peut se perdre par excès ou par défaut: par l'esprit d'égalité, qui devient " extrême "; par l'esprit d'inégalité, qui produit lui aussi la dégénérescence du corps politique. La vertu se perd par excès d'égalité, lorsque le désir d'égalité devient désir de nivellement et refus de toute hiérarchie (VIII, 2). Or l'esprit d'égalité ne consiste pas à faire que tout le monde commande, mais "à obéir et à commander à ses égaux ". Montesquieu est formel: la démocratie se dérègle lorsque les hommes ne veulent plus seulement être égaux en tant que citoyens, mais aussi « comme magistrat, comme sénateur, comme juge, comme père, comme mari, comme maître " (VIII, 3). Mais la vertu se perd également par défaut d'égalité et de frugalité, avec l'introduction du luxe et du désir de jouissances:

«À mesure que le luxe s'établit dans une république, l'esprit se tourne vers l'intérêt particulier. À des gens à qui il ne faut rien que le nécessaire, il ne reste à désirer que la gloire de la patrie et la sienne propre. Mais une âme corrompue par le luxe a bien d'autres désirs: bientôt elle devient ennemie des lois qui la gênent» (VII, 2).

La corruption intervient lorsque l'éducation ne suffit plus à contrer les tendances égoïstes de l'homme: les valeurs publiques perdent alors leur sens au regard de nouveaux critères, individualistes, du jugement moral (III, 3). L'Esprit des lois en conclut à la nécessité de ramener périodiquement la république à ses principes, c'est-à-dire, comme l'avait vu Machiavel ${ }^{8}$, de restaurer les coutumes anciennes qui pérennisent la vertu (VIII, 12).

La conséquence en est que la démocratie et la vertu sont hors de portée de la plupart des peuples modernes. Seule l'égalité fait aimer l'égalité, seule la frugalité généralisée permet de ne pas faire surgir le goût du luxe. Pour cela, il faut impérativement de petits États, homogènes, simples, égalitaires, frugaux. La condition portant sur la morphologie du territoire est particulièrement stricte. Pour Montesquieu, l'ethos démocratique ne peut être préservé que dans un État de petites dimensions, où le bien commun est visible et proche de chaque citoyen:

«Il est de la nature de la république qu'elle n'ait qu'un petit territoire; sans cela elle ne peut guère subsister. Dans une grande république il y a de grandes fortunes, et par conséquent peu de modération dans les esprits [...]; les intérêts se particularisent; un homme sent d'abord qu'il peut être heureux, grand, glorieux, sans sa patrie; et bientôt qu'il peut être seul grand

8. Sur le rapport ambivalent de Montesquieu à Machiavel, voir Spector C., " Montesquieu ou les infortunes de la vertu ", Esprit, numéro sur "La corruption, maladie de la démocratie ", n 402 , Février 2014, p.31-44. 
sur les ruines de sa patrie. Dans une grande république, le bien commun est sacrifié à mille considérations; il est subordonné à des exceptions; il dépend des accidents. Dans une petite, le bien public est mieux senti, mieux connu, plus près de chaque citoyen; les abus y sont moins étendus, et par conséquent moins protégés (VIII, 16).»

La vertu politique est donc exclue, pour l'essentiel, dans les grands États. Du moins devra-t-on apprendre à pouvoir se passer d'elle, comme l'ont appris à leurs dépens les Anglais sous Cromwell (III, 3). Tout autant, le développement économique, qui entraîne le plus souvent l'accroissement des richesses et des inégalités, semble exclure la vertu des temps modernes, à l'exception de quelques frugales républiques commerçantes, comme la Hollande du siècle précédent, ou Genève et quelques cantons suisses ${ }^{9}$. En général, l'opposition entre vertu et commerce est irréductible. Rousseau s'en souviendra:

«Les politiques grecs, qui vivaient dans le gouvernement populaire, ne reconnaissaient d'autre force qui pût les soutenir que celle de la vertu. Ceux d'aujourd'hui ne nous parlent que de manufactures, de commerce, de finances, de richesses et de luxe même" (III, 3).

La corruption des mœurs menace ainsi la modernité tout entière. Montesquieu va jusqu’à évoquer l'exception des jésuites du Paraguay « dans la lie et la corruption des temps modernes" (IV, 6). Il évoque les « institutions singulières " des républiques anciennes qui semblent si étranges au regard des modernes (IV, 8). À cet égard, Montesquieu ne propose nullement d'universaliser les principes de la vertu sacrificielle. Dans les grands États modernes, l'art politique fonctionne à l'honneur, ou pour le dire autrement, au désir de reconnaissance:

« L'État subsiste indépendamment de l'amour pour la patrie, du désir de la vraie gloire, du renoncement à soi-même, du sacrifice de ses plus chers intérêts, et de toutes ces vertus héroïques que nous trouvons dans les anciens, et dont nous avons seulement entendu parler» (III, 5) ${ }^{10}$.

\section{De Montesquieu à Robespierre : vertu et terreur}

Or Robespierre réinvestit et subvertit les principes issus de L'Esprit des lois. Des travaux ultérieurs devraient examiner le magistère de Rousseau, et son importance dans la prise de distance de l'Incorruptible à l'égard de son premier mentor: la théorie de la corruption comme prévalence de la volonté

9. Voir Spector C., Montesquieu. Pouvoirs, richesses et sociétés, Paris, PUF, 2004 (rééd. Hermann, 2011); Montesquieu et l'émergence de l'économie politique, Paris, Honoré Champion, 2006.

10. Sur l'honneur, voir Spector C., Montesquieu. Pouvoirs, richesses et sociétés, Paris, PUF, 2004, rééd. Hermann, 2011, chap. 1 . 
particulière sur la volonté générale joue ici un rôle majeur ${ }^{11}$. En l'an II, Robespierre hérite en effet de la redoutable difficulté identifiée par Montesquieu et par Rousseau (le risque de corruption de la vertu, qui entraîne la république à sa perte), dans le contexte inédit en France de l'instauration d'une république souveraine dans un grand État - ce pourquoi il évoque le " principe du gouvernement démocratique ou populaire». Et contrairement à Montesquieu, Robespierre ne croit pas à la solution de la monarchie constitutionnelle à l'anglaise ou à l'équilibre des pouvoirs " faible image de la liberté » qui a pu en duper certains aux débuts de la Révolution. Seule la république démocratique, associée à la souveraineté populaire, peut être légitime. Reste un problème majeur: les magistrats, qui ne sont que les commissaires du peuple, risquent toujours de se détourner du bien public. C'est ce que suggère Robespierre dans son discours du 17 pluviôse :

«La vertu républicaine peut être considérée par rapport au peuple, et par rapport au gouvernement: elle est nécessaire dans l'une et dans l'autre. Quand le gouvernement seul en est privé, il reste une ressource dans celle du peuple; mais, quand le peuple lui-même est corrompu, la liberté est déjà perdue.

Heureusement, la vertu est naturelle au peuple, en dépit des préjugés aristocratiques...

D'ailleurs, on peut dire, en un sens, que pour aimer la justice et l'égalité, le peuple n'a pas besoin d'une grande vertu; il lui suffit de s'aimer lui-même.

Mais le magistrat est obligé d'immoler son intérêt à l'intérêt du peuple, et l'orgueil du pouvoir à l'égalité. Il faut que la loi parle surtout avec empire à celui qui en est l'organe ${ }^{12}$."

Avant Rousseau, Montesquieu avait d'ores et déjà souligné que la corruption, en démocratie, commence rarement par le peuple, et tient d'abord aux élites $(\mathrm{V}, 2)$. Il avait refusé d'identifier la corruption aux prémisses de la rébellion, et avait même justifié, pour la Crète antique, le principe de l'insurrection populaire:

«Les Crétois, pour tenir les premiers magistrats dans la dépendance des lois, employaient un moyen bien singulier: c'était celui de l'insurrection. Une partie des citoyens se soulevait ${ }^{13}$, mettait en fuite les magistrats, et les obligeait de rentrer dans la condition privée. Cela était censé fait en conséquence de la loi (VIII, 11).»

11. Claude Mazauric a étudié en détail la « Dédicace aux mânes de Jean-Jacques Rousseau " rédigée par Robespierre (« Maximilien Robespierre dans l’ombre vivante de Jean-Jacques Rousseau », dans Michel Biard et Philippe Bourdin [dir.], Robespierre. Portraits croisés, Paris, Armand Colin, 2013, p. 23-50).

12. Rapport du 17 pluviôse an II-5 février 1794, OC, t.X, p. 355-356.

13. Aristote, Politique, liv. II, chap.X [note de Montesquieu]. 
Robespierre en tire les conséquences dans la situation nouvelle de la France; pour aimer la justice et l'égalité, le peuple n'a pas besoin de vertu sacrificielle, puisqu'il profite immédiatement de leurs bienfaits. Aussi ne doit-on que le protéger de ses ennemis et de ses oppresseurs. Pour imposer la révolution contre les ennemis de l'intérieur et les coalitions de l'extérieur, la morale politique doit désormais l'emporter: "Dans le système de la Révolution française, ce qui est immoral est impolitique, ce qui est corrupteur est contre-révolutionnaire ${ }^{14}$."

Robespierre hérite ainsi de Montesquieu, de Rousseau et même de Machiavel, leur grand maître commun, dans sa hantise de la corruption. Comme Montesquieu, il accepte que le principe de la république démocratique puisse se corrompre par excès ou par défaut (aristocratie de ceux qui gouvernent, mépris du peuple pour les autorités qu'il a lui-même établies). Mais la situation qu'il doit affronter est totalement nouvelle: une république dans un grand État - ce que ni les uns ni les autres ne croyaient possible. Une république nouvelle doit émerger du terreau corrompu de l'Ancien Régime, et donc régénérer la nation. Robespierre ne dispose plus de l'atoutmaître de Montesquieu, qu'il héritait lui-même de Machiavel: le "retour aux principes ». Dans la situation révolutionnaire, le " retour " aux principes est désormais impossible. Il faut donc exclure, après les aristocrates, les traîtres, les intrigants et les «faux patriotes » qui entendent devenir la Nomenklatura du Nouveau régime. Comme Montesquieu et comme Rousseau, Robespierre compte sur la vertu du peuple plus que sur celle des magistrats :

«Le peuple veut toujours le bien public, parce qu'il est le peuple; il n'a pas même besoin de vertu, pour être juste; car c'est à lui-même qu'il rend justice ${ }^{15}$."

Mais contrairement à ses prédécesseurs, Robespierre doit lutter contre les circonstances hostiles qui le conduisent à proposer une alliance inouïe, un mariage contre-nature: l'alliance de la vertu et de la terreur. Dans L'Esprit des lois, la crainte était réservée au seul despotisme - ce que Robespierre rappelle ("On a dit que la terreur était le principe du gouvernement despotique »), mais pour mieux marquer la différence des situations, qui déjoue les faux-semblants:

«Le vôtre [de gouvernement] ressemble-t-il donc au despotisme? Oui, comme le glaive qui brille dans les mains des héros de la liberté ressemble à celui dont les satellites de la tyrannie sont armés [...] Domptez par la terreur les ennemis de la liberté, et vous aurez raison, comme fondateurs de la République ${ }^{16}$.»

14. $O C, \mathrm{t} . \mathrm{X}, \mathrm{p} .353$

15. $O C$, t. V, p. 200.

16. $O C$, t. X, p. 274,354 . 
C'est ici que Robespierre prend congé de Montesquieu. Substituer la morale à l'égoïsme, la probité à l'honneur, l'amour de la patrie et de la gloire à la cupidité, les vertus de la république aux vices et aux ridicules de la monarchie, tout cela ne peut se faire sans peine: fonder et consolider la démocratie, parvenir au règne paisible des lois, suppose de "terminer la guerre de la liberté contre la tyrannie " et de " traverser heureusement les orages de la révolution » dans les circonstances tragiques que la Révolution rencontre. Partout des traitres à démasquer, partout des émissaires soudoyés par l'étranger. Après avoir cru que la vertu pouvait être spontanée, Robespierre en vient à défendre le peuple contre lui-même, et contre les conspirateurs et calomniateurs qui le pervertissent. Parce que la démocratie est toujours en péril, comme l'avait vu Montesquieu, la tâche des représentants du peuple est de faire justice à ceux qui tentent de les avilir. L'alternative tragique devient celle de la servitude ou de la guerre civile. D’où la nécessité d'ordonner les maximes politiques, durant cette période transitoire, à la révolution plutôt qu'à la démocratie. D'où la tentation du «despotisme de la liberté » et de l'application rigoureuse de cette " morale politique », fraternelle et fratricide, dont Montesquieu nous avait appris à nous méfier et dont Robespierre n'a douté, tragiquement, qu'à la veille de sa mort ${ }^{17}$. 